\title{
Validación de una Matriz para Evaluar Monografías en Estudiantes Universitarios
}

\author{
Gloria M. Mora(1), Loreto A. Riquelme ${ }^{(1)}$, Jorge C. Troncoso ${ }^{2)}$ y Bertha A. Escobar ${ }^{(1)}$ \\ Universidad Católica de Temuco, (1) Carrera de Psicología, (2) Carrera de Antropología, Manuel Montt 056, \\ Temuco-Chile (e-mail: gmora@uct.cl; Iriquelme@uct.cl; jtroncoso@uct.cl; bescobar@uct.cl)
}

Recibido Abr. 28, 2017; Aceptado Jun. 27, 2017; Versión final Sep. 1, 2017, Publicado Feb. 2018

\begin{abstract}
Resumen
El trabajo se propuso establecer las propiedades psicométricas de una matriz de valoración de las competencias gestión del conocimiento y pensamiento crítico por medio de la elaboración de una monografía de compilación por estudiantes de primer año de la Carrera de Psicología de la Universidad Católica de Temuco en Chile. Se empleó el método de agregados individuales para estimar la validez de contenido del instrumento diseñado, mientras que con los datos generados a partir de la revisión de 69 monografías se estimó la consistencia interna del instrumento mediante el uso del estadígrafo Alfa de Cronbach. Adicionalmente, se obtuvo el Índice de Discriminación de cada criterio. Los resultados muestran que la matriz trabajada es un instrumento válido y confiable. Se muestra que la matriz garantiza una evaluación útil al estudiante que le permite seguir su avance en el desarrollo de la gestión del conocimiento y el pensamiento crítico.
\end{abstract}

Palabras clave: propiedades psicométricas; matriz de valoración; gestión del conocimiento; pensamiento crítico; pregrado

\section{Validation of a Rubric to Evaluate Monographs in University Students}

\begin{abstract}
The purpose of this study was to establish the psychometric properties of an evaluation rubric of knowledge management and critical thinking competencies by means of the elaboration of a compendium monograph by freshman students of the Career of Psychology of the Catholic University of Temuco in Chile. The individual aggregates method was used to estimate the content validity of the designed instrument, while the data generated from the review of 69 monographs estimated the internal consistency of the instrument using the Cronbach Alpha statistical parameter. Additionally, the Discrimination Index was obtained for each criterion. The results show that the rubric worked is a valid and reliable instrument. It is shown that the rubric guarantees a useful evaluation for the students allowing them progressing in the development of knowledge management and critical thinking competencies.
\end{abstract}

Keywords: psychometric properties; evaluation rubrics; knowledge management; critical thinking; undergraduate 


\section{INTRODUCCIÓN}

El campo de la educación se ha transformado radicalmente en las últimas décadas quedando atrás los sistemas educativos tradicionales que funcionaban con base en la edad, para pasar a la educación que forma al estudiante a aprender a aprender, lo que implica que él o ella adquiera una serie de competencias, tales como el pensamiento crítico y la gestión del conocimiento, con las cuales pueda adaptarse al ámbito profesional (Reimers y Chung, 2016). Esta forma de formación universitaria se ha llamado modelo educativo basado en competencias (Blanco, 2011).

Desde el año 2008, la Universidad Católica de Temuco (UC Temuco) ha implementado el modelo educativo basado en competencias (UC Temuco, 2007), entendiendo a las competencias como procesos complejos de desempeño donde se integra el ser, el saber y el saber hacer. En la UC Temuco, los programas distinguen entre competencias específicas, o aquellas que reflejan un desempeño propio de la disciplina, y competencias genéricas, o aquellas que permiten el desarrollo de cada estudiante en su dimensión intra e interpersonal (UC Temuco, 2007). Las competencias genéricas se incorporan de manera explícita en las asignaturas del currículum las que deben mostrar coherencia entre los resultados de aprendizaje, las tareas y los procesos evaluativos (Biggs y Tang, 2007). Lograr tal coherencia exige al docente actualizarse de manera permanente tanto en la enseñanza como en la evaluación de las competencias genéricas (Villarroel y Bruna, 2014).

En el modelo basado en competencias, la evaluación es de gran relevancia porque se constituye en una herramienta objetiva que funciona como apoyo para impulsar el trabajo continuo y progresivo del estudiante, a través de la valoración de su desempeño en diversas situaciones o tareas auténticas (Fernández 2010). Una evaluación de este tipo se puede implementar por medio de matrices de valoración o rúbricas (Manríquez, 2012). Las matrices de valoración son instrumentos de evaluación que proporcionan retroalimentación formativa al estudiante permitiéndole tener claro lo que se espera que realice, lo que ha logrado y lo que debe mejorar (Atkinson y Lim, 2013; Lim, 2013). No obstante la utilidad de las matrices de valoración, para que éstas estimen de manera válida y confiable los desempeños logrados por el estudiante, deben pasar por un proceso previo en el que se establezcan sus propiedades psicométricas lo que permite garantizar su validez y confiabilidad.

Atendiendo a esta problemática, el objetivo del presente estudio fue determinar la validez y la confiabilidad de una matriz para evaluar las competencias gestión del conocimiento y pensamiento crítico en estudiantes de la carrera de Psicología de la UC Temuco. La carrera de Psicología ha definido entre otras a la competencia genérica gestión del conocimiento y a la competencia específica pensamiento crítico como competencias básicas en su itinerario formativo. En cuando a la gestión del conocimiento, ésta se puede definir como un proceso dinámico e interactivo de intercambio de información, que puede ser de carácter personal o colectivo y que permite generar aprendizajes activos por medio del compartir ideas y extender los propios puntos de vista al considerarlos desde diferentes perspectivas (Gairín, 2007). En la carrera, el documento institucional Competencias Genéricas UC Temuco: Para la Formación Integral de Ciudadanos Socialmente Responsables (UC Temuco, 2008) define la gestión del conocimiento como aquella competencia que permite al estudiante procesar información para la generación de conocimiento por medio de conocer, comprender, aplicar, analizar, sintetizar, evaluar y transformar la información de acuerdo a las necesidades de aprendizaje y del trabajo, así como de las exigencias del medio sociocultural.

En relación al pensamiento crítico, Blanco (2011) revisa las conceptualizaciones de autores de importante renombre en el área como Peter Facione, Carlos Saiz, Ana María Nieto, Richard Paul y Linda Elder, para explicar que el pensamiento crítico consiste en una competencia donde la persona puede expresar una opinión particular sobre un hecho, situación o información de manera fundamentada, a través de argumentos que la sustentan. Esto supone haber identificado diferentes puntos de vista con respecto a la problemática, haberlos analizado y establecido criterios que le permitan emitir un juicio de valor (Blanco, 2011). A nivel de la carrera de Psicología, la competencia de pensamiento crítico se ha entendido como la comprensión que hace el estudiante de los procesos psicosociales que involucran a las personas y los colectivos por medio de analizar las tensiones sociales implicadas en los distintos niveles de organización social y con la finalidad de tomar decisiones orientadas a generar procesos de cambio (Mora, 2015). En esta aproximación se evidencian algunos de los aspectos expuestos por Blanco (2011), entre ellos, el uso de argumentos para tomar decisiones fundamentadas en lo ético, la responsabilidad social y la intelectualidad.

Con base en las anteriores orientaciones conceptuales, el objetivo de este estudio fue establecer las propiedades psicométricas de una matriz de desempeño utilizada en estudiantes de primer año de la carrera de Psicología para evaluar las competencias gestión del conocimiento y pensamiento crítico a través de la elaboración de una monografía de compilación que forma parte de las evidencias de aprendizaje de la asignatura Fundamentos Filosóficos de las Ciencias Sociales. Según Corona (2015), la monografía de 
compilación consiste en una investigación documental que realiza el estudiante a partir de la que integra, organiza y evalúa la información teórica y empírica existente sobre un problema y la presenta de forma crítica. Implica, por una parte, que el estudiante exponga los diferentes puntos de vista sobre el tema investigado y, por otra, que muestre su opinión personal sobre los mismos (Corona, 2015). El estudio fue relevante porque permitió validar un instrumento que puede ocuparse a partir de ahora de manera confiable en situaciones de aprendizaje similares. Asimismo, fue relevante porque generó evidencia sobre los mecanismos que se siguen en la carrera para garantizar el aprendizaje de los estudiantes, lo que constituye uno de los requerimientos que se evalúa en los procesos de acreditación de los programas cuyo modelo educativo es el modelo basado en competencias (Atkinson y Lim, 2013; Muñoz y Valenzuela, 2015).

\section{MATRIZ DE VALORACIÓN}

La matriz de valoración puede entenderse como una guía o pauta de evaluación auténtica sobre el desempeño del estudiante, que funciona con base en criterios de valoración y diferentes niveles de logro progresivo con respecto a la ejecución de una tarea (Raposo y Martínez, 2011). La matriz de valoración tiene la finalidad de explicitar al estudiante lo que se espera de él o ella, de valorar su desempeño y de ser un instrumento de retroalimentación del mismo (Gatica y Uribarren, 2012; Fernández, 2010; Manríquez, 2012). Por estas propiedades es que los estudiantes perciben las matrices de valoración especialmente útiles para clarificar los requisitos que deben cumplir sus trabajos, centrar la atención en los criterios a considerar, facilitar una autoevaluación realista y mejorar la calidad de la retroalimentación que reciben de parte del docente u otros pares, además de que ellos consideran que las matrices les orientan más hacia las metas de aprendizaje (García-Ros, 2012; Prins et al., 2015; Panadero et al., 2014).

Respecto al proceso de elaboración de una matriz de valoración, en términos generales éste consiste en diseñar un instrumento que sea capaz de medir estructuras psicológicas que generalmente no pueden ser observadas de manera directa en el comportamiento del estudiante (Gürsul y Keser, 2009). De acuerdo a Guzmán et al. (2012) existen tres formas de elaborar una matriz de valoración. En la primera, los indicadores de distintos niveles de desempeño se analizan y modifican en conjunto con los usuarios y posteriormente se prueban con distintos evaluadores. En la segunda, se comienza con la entrevista a muestras independientes de estudiantes que reflejen la respuesta que se desea calificar; en estas entrevistas se identifican patrones de respuesta de los estudiantes consistentes e inconsistentes y, posteriormente, se discuten y reconcilian las respuestas inconsistentes para repetir el proceso de medidas independientes en nuevas muestras de estudiantes. En la tercera, el diseño y uso de la rúbrica por parte de expertos se inicia buscando ejemplos en los que se identifican características de desempeño: se hace una lista de los criterios que demuestran calidad, se articulan de manera gradual y se califican los ejemplos para comprobar la capacidad discriminante; se usa la rúbrica con trabajos nuevos para valorar si discrimina niveles de calidad. Esta última forma también puede llamarse de validación de contenido por parte de jueces expertos, quienes determinan si los componentes y la rúbrica son pertinentes para evaluar los productos elegidos (Guzmán et al., 2012). Al respecto, según Reddy y Andrade (2010) una matriz de valoración bien diseñada debería reducir las inconsistencias en los procesos de valoración inter e intra juez.

En el caso específico de la evaluación de las competencias gestión del conocimiento y pensamiento crítico, hay evidencias respecto a que una forma de evaluar las mismas es a través de producciones escritas, con la consecuencia de que en este caso se requiere contar con una matriz a partir de la cual valorar las características de las argumentaciones para diferenciar argumentos bien construidos de aquellos pobremente construidos (Lu y Zhang, 2013). Para lograr esto, la matriz debe focalizarse en las siguientes dos dimensiones: una, la global, que refiere a los aspectos organizativos y de estructura del texto considerado en su conjunto; y, otra, la local, que alude a aspectos referidos a elementos específicos del texto tales como la conexión entre frases y la ortografía (Muñoz y Valenzuela, 2015).

Ambas dimensiones deben evaluarse de manera analítica, es decir, desglosando los componentes del desempeño del estudiante para luego obtener una calificación global, lo que favorece la retroalimentación efectiva al estudiante sobre su desempeño (Gatica y Uribarren, 2012). Con base en estos antecedentes, el estudio se propuso el diseño de una matriz de valoración para producción escrita tipo monografía de compilación, la cual es una evidencia de aprendizaje a partir de la cual acceder al desarrollo de la gestión del conocimiento y el pensamiento crítico en el estudiante. En el siguiente apartado se presenta la metodología empleada para estimar la confiabilidad y validez del instrumento desarrollado en el marco de la carrera de Psicología de la UC Temuco.

\section{METODOLOGÍA}

El trabajo realizado tuvo el propósito de diseñar una matriz de valoración válida y confiable para evaluar el desempeño de estudiantes de primer grado en relación a las competencias gestión del conocimiento y 
pensamiento crítico a través de elaborar una monografía de compilación, la cual forma parte de las evidencias de aprendizaje de la asignatura Fundamentos Filosóficos de las Ciencias Sociales de la carrera de psicología de la UC Temuco. Como parte de los resultados de aprendizaje de este curso, el estudiante debe dar cuenta de las principales características de los tres grandes paradigmas de las Ciencias Sociales (positivista, fenomenológico-interpretativo y sociocrítico), a la vez que identificar algunos problemas epistemológicos propios del campo y tomar una postura epistemológica, ética y/o política como futuro profesional ante los mismos (Mora, 2015). Como evidencia de aprendizaje, la asignatura contempla la elaboración de una monografía de compilación a través de la cual se evalúan las competencias genérica y específica del curso, respectivamente, gestión del conocimiento y pensamiento crítico. El estudiante cuenta con la guía presentada en la tabla 1 para elaborar su monografía. La elaboración de esta guía se basó en los lineamientos que establece Corona (2015) respecto a la monografía de compilación; este tipo de monografía debe partir de la elección de un tema, para posteriormente analizarlo y redactar una presentación crítica del mismo con base en la bibliografía y otras referencias consultadas (Corona, 2015).

Tabla 1: Detalles sobre la guía para elaboración de monografía

Guía para elaboración de monografía de compilación

Objetivo de aprendizaje: Escribir una monografía de compilación donde se presenten los resultados de una revisión bibliográfica respecto de un tema particular, siendo la información presentada de una forma organizada y que incluye un punto de vista crítico del autor/a. Para los fines de esta guía y su correspondiente matriz de evaluación se entiende por crítico reconocer posibilidades y límites, así como convergencias y disimilitudes entre las aproximaciones epistemológicas y sus efectos en distintos ámbitos de aplicación.

Para los fines del curso Fundamentos Filosóficos de las Ciencias Sociales, deben considerarse los siguientes lineamientos:

I. Tema: Éste debe formar parte de las reflexiones y propuestas epistemológicas de la Ciencias Sociales en cualquiera de sus tradiciones.

II. Género: Se trata de una monografía de compilación.

III. Modalidad: Individual.

IV. Apartados básicos y requerimientos solicitados para cada uno:

1. Portada. Contiene el nombre de la institución, del ramo, de la docente y del alumno/a, así como la fecha de entrega.

2. Índice. Presenta una tabla de los apartados y subapartados del trabajo.

3. Resumen y palabras clave: El resumen constituye una síntesis del trabajo, incluyendo el problema que se analiza, una breve descripción de los criterios de elección bibliográfica elegidos, los principales resultados y las conclusiones más relevantes. No debe ser de más allá de 150 palabras. Adicionalmente, se deben poner un máximo de 5 palabras claves.

4. Introducción. Explica el propósito del trabajo, la problemática estudiada, el enfoque del autor/a y las limitaciones del estudio. Se expone brevemente en qué consiste todo el documento.

5. Cuerpo del trabajo. Se presenta información organizada respecto al tema que se trabajó. Cada autor/a define los subapartados más adecuados para el desarrollo de su investigación bibliográfica sobre el tema.

6. Conclusión. Se argumenta en relación a cómo el trabajo respondió a los objetivos iniciales. Se enuncian las implicaciones del estudio en el ámbito científico.

7. Referencias. Se citan a los autores y autoras referenciados en el trabajo, siguiéndose las normas APA $6^{a}$ edición.

8. Anexos (opcional).

V. Criterios formales: Letra Arial o Times New Roman número 12, interlineado 1,5; extensión requerida: entre 10 a 12 páginas (no incluye portada, índice, bibliografía ni anexos); y, márgenes $2,5 \mathrm{~cm}$ superior, inferior, izquierdo y derecho.

VI. Criterios de aceptación de bibliografía como fuente reconocida: Se aceptarán como fuentes reconocidas los siguientes: a) artículos publicados en revistas indexadas en bases Scopus, Latindex, Scielo, Dialnet, EBSCOHost, JSTOR, Web of Science, o bien, que cuentan con un proceso de arbitrio de artículos previo a su publicación; y, b) libros, capítulos de libros o informes que forman parte de la tradición disciplinar.

El procedimiento de validación de la matriz diseñada se inició con una revisión bibliográfica sobre experiencias y/o instrumentos similares en el ámbito de la evaluación de la gestión del conocimiento y el pensamiento crítico. Esta primera etapa se llevó a cabo durante el primer semestre académico del 2015 y en ella se definieron los niveles de logro esperados para el estudiante de primer año en relación a su 
desarrollo de las competencias de interés. En gestión del conocimiento, el nivel de logro se definió como la competencia del estudiante para "reconoce[r] las habilidades y/o procesos cognitivos con los cuales puede identificar, seleccionar, relacionar e interpretar información básica relacionada con su profesión futura” (UC Temuco, 2008, p. 30). En cuanto al pensamiento crítico, el nivel de logro se definió como la competencia del estudiante para "conoce[r] y comprende[r] los distintos tipos de miradas posibles para la explicación de un fenómeno psicológico individual y/o colectivo, siendo capaz de diferenciar aquellos juicios que surgen de su propia experiencia de aquellos basados en evidencia conceptual, empírica o contextual” (Mora, G. 2015).

Con base en las anteriores definiciones de niveles de logro, se establecieron los siguientes criterios evaluativos para la matriz de valoración de una monografía de compilación: 1) Formato y presentación, refiere a si el trabajo se ajusta a los lineamientos formales y estructurales solicitados en la guía de elaboración; 2) Organización, alude a la identificación de apartados y subapartados, con sus títulos y subtítulos respectivos dentro del trabajo, así como el nivel del logro en la articulación entre los mismos; 3) Referencias bibliográficas, corresponde al número y la calidad de las fuentes bibliográficas consultadas; 4) Autoría, atañe a la construcción de una propuesta de monografía desde el punto de vista personal del autor/a y con base en una sólida argumentación; y, 5) Logro del trabajo, que representa el logro general del producto solicitado, esto es, una monografía de compilación.

Tanto la Guía para elaboración de una monografía de compilación como la matriz de valoración originalmente diseñada para la evaluación de la gestión del conocimiento y el pensamiento crítico a partir de la monografía, se usaron durante el curso de Fundamentos Filosóficos de las Ciencias Sociales del año 2015. Cabe señalar que en este momento la aplicación de la matriz de valoración se realizó sin conocer las propiedades psicométricas de la misma; de hecho, el proceso de obtención de estas propiedades se realizó de forma paralela y posterior al cierre del curso. La intención era contar con un instrumento válido y confiable para ser usado en las siguientes versiones de la asignatura.

Una vez diseñada la matriz de valoración original se estimó su validez de contenido, entendido como el grado en que los criterios y niveles de un instrumento son representativos del universo de contenido que se quiere medir (Corral, 2009), en este caso, las competencias gestión del conocimiento y pensamiento crítico, ambas en los niveles de logro previamente definidos como esperables para un estudiante de primer año. Siguiendo el método de agregados individuales (Corral, 2009), se inició la etapa de selección de expertos para ajustar o revisar los conceptos presentes en el instrumento así como la propuesta de valoración asociada a cada uno de ellos. Los criterios para la selección de los expertos fueron contar con experticia profesional en educación superior y en procesos de evaluación basado en competencias del desempeño del estudiante. El número de expertos seleccionados correspondió a tres, quienes recibieron la matriz de valoración diseñada y su guía de referencia junto a una pauta de revisión que fue devuelta con comentarios. La pauta de revisión incluía la estimación de los criterios incluidos en el instrumento en relación a su claridad en la redacción, coherencia interna, nivel de lenguaje adecuado al nivel del estudiante, pertinencia, valoración o ponderación y distinción de niveles de desempeño. Las observaciones de los jueces expertos fueron sistematizadas y analizadas por el equipo investigador, tomándose decisiones respecto a su incorporación o no en la matriz de valoración.

Posteriormente, se realizó una segunda selección de jueces constituida por una muestra intencionada de cuatro pares docentes con experiencia en docencia de pregrado de cursos de la línea de formación epistemológica en las Ciencias Sociales. A los pares docentes se les entregó la matriz de valoración diseñada y su guía de referencia; en este momento, la matriz de valoración ya había sido modificada conforme a la revisión primera basada en los comentarios entregados por los jueces expertos. También a los pares docentes se les hizo entrega de la misma pauta de revisión que usaron los jueces expertos y también ellos la devolvieron con comentarios. Se pidió a los pares docentes que enfatizaran su opinión sobre la matriz en términos de su calidad evaluadora. Durante esta fase se contó con los aportes de tres pares evaluadores, porque se descartaron los comentarios del cuarto evaluador, ya que éste no se ajustó a la pauta de evaluación entregada ni tampoco emitió opinión pertinente al procedimiento seguido. Los resultados de esta etapa fueron nuevamente sistematizados e incorporados de acuerdo a su pertinencia. De esta manera se construyó la matriz de valoración final, la cual se presenta en la Tabla 2.

Con la matriz de valoración final, se realizó la tercera etapa del proceso la cual consistió en una prueba piloto constituida por una muestra intencionada de cuatro docentes que evaluaron una experiencia real con el instrumento de valoración diseñado y ajustado a partir de los expertos y pares. Para ello, se entregó a cada docente el instrumento y se le solicitó que con el mismo evaluaran el desempeño que habían logrado los estudiantes de la asignatura Fundamentos Filosóficos de las Ciencias Sociales. Los docentes evaluaron 14, 19, 17 y 19 monografías respectivamente, haciendo un total de 69 evidencias de aprendizaje, las cuales correspondían a las monografías que habían entregado los estudiantes que cursaron la asignatura el 
semestre anterior, esto es, durante el primer semestre del año 2015. Los puntajes asignados por criterio a cada monografía podían ser 4 para el nivel de logro sobresaliente; 3,5 ó 3 para avanzado; 2,5 ó 2 para suficiente; 1,5 ó 1 para insuficiente o 0 para no evidencia. Los resultados fueron recogidos e incorporados en una base de datos que se diseñó en el paquete estadístico SPSS 22.0. Con estos datos, se realizó una prueba de confiabilidad interna a través del estadígrafo Alfa de Cronbach el cual permite cuantificar la correlación entre los ítems que componen el instrumento (Oviedo y Campo, 2005) y es utilizada cuando se trata de alternativas de respuestas policotómicas (Corral 2009; Hernández et al., 2008). Adicionalmente, se obtuvo el Índice de Discriminación de cada criterio, así como una estimación del nivel de dificultad de los criterios evaluados en la matriz de valoración. Los resultados y la discusión del proceso se presentan en el apartado que sigue.

\section{RESULTADOS}

Respecto a la estimación de la validez de contenido se encontró que los cinco criterios de la matriz diseñada para la valoración de las competencias gestión del conocimiento y pensamiento crítico a través del desempeño mostrado por el estudiante en una monografía de compilación, fueron estimados como pertinentes y coherentes internamente tanto por la totalidad de los jueces expertos como la totalidad de los pares docentes. Ambos grupos de evaluadores, hicieron sugerencias respecto a precisar la distinción de ciertos niveles de desempeño, clarificar la redacción de algunos o modificar la ponderación de los criterios.

Respecto a las observaciones realizadas por los jueces expertos, éstas incluyeron incorporar en la descripción de los niveles de logro los descriptores coherencia y cohesión del texto en Organización y uso de las reglas ortográficas del idioma en Formato y presentación, así como subir la ponderación de este último criterio de un $10 \%$ a un $15 \%$. No obstante, la coincidencia respecto a estas tres observaciones fue parcial, por lo que siguiendo a Corral (2009), las mismas se incorporaron, pero se sometieron a una segunda etapa de validación, la cual consistió en la valoración por pares docentes. A fin de subir la ponderación para el criterio Formato y presentación, se bajó la ponderación de Logro del trabajo de un $25 \%$ inicial a un $20 \%$, lo que también se sometió a la evaluación de los pares docentes. Otras observaciones respecto a mejorar la redacción de los contenidos de la matriz fueron incorporadas.

Por otra parte, en esta fase se rechazaron las observaciones provenientes de uno de los jueces en relación a bajar la ponderación correspondiente a los criterios Organización y Referencias bibliográficas y, en cambio, aumentar a un $30 \%$ la ponderación de la Autoría y a otro $30 \%$ la correspondiente al Logro del trabajo. La decisión se tomó considerando que la matriz de valoración se dirigió a estudiantes de primer año del pregrado que para desarrollarse en gestión del conocimiento y pensamiento crítico deben en primer término desarrollar su habilidad para organizar la información, así como para buscar fuentes de calidad para su trabajo. Esta decisión, no obstante, también quedó sujeta a la evaluación posterior que hicieran los pares docentes.

En cuanto a la estimación de la validez de contenido por los pares docentes, además de la coincidencia total respecto a la pertinencia y coherencia interna de los cinco criterios de la matriz diseñada, los tres manifestaron coincidencia respecto a las modificaciones incorporadas al instrumento a partir de las observaciones hechas por los jueces expertos. Los pares docentes sugirieron aumentar aún más la ponderación del criterio Formato y presentación argumentando que, por tratarse de estudiantes de primer año, una de las principales tareas del docente es fomentar en ellos su habilidad para ajustarse a los requerimientos formales; en concreto, un par docente sugirió ponderar este criterio con un 20\%. De igual modo, en Formato y presentación los pares docentes coincidieron en detallar con mayor precisión los descriptores en cada nivel de logro, lo que permitiría distinguir los mínimos y los máximos de desempeño en cada nivel. Estas observaciones fueron incorporadas en su totalidad al instrumento.

Así mismo, entre los pares docentes hubo coincidencia respecto a la necesidad de operacionalizar más algunos descriptores a fin de distinguir con más precisión lo que se espera del estudiante por nivel de logro. Estas observaciones fueron incorporadas, sobre todo en los criterios Referencias bibliográficas y Autoría. Al respecto, se eliminó el descriptor originalidad del problema de investigación, el cual se incluía en el criterio Autoría en la matriz original, esto debido a que los pares docentes coincidieron que la originalidad no constituye un desempeño esperable para un estudiante del primer año. Por último, para subir en un $5 \%$ el porcentaje de ponderación de Formato y presentación, se restó $5 \%$ a la ponderación del Logro del trabajo. La razón de esta última decisión se basó en la sugerencia de un par docente respecto a que el criterio Logro del trabajo alude a una habilidad de síntesis global, por lo que debe tener una ponderación máxima de 15\%, privilegiándose otros criterios que implican un mayor desglose de los componentes del desempeño del estudiante (Gatica y Uribarren, 2012). 


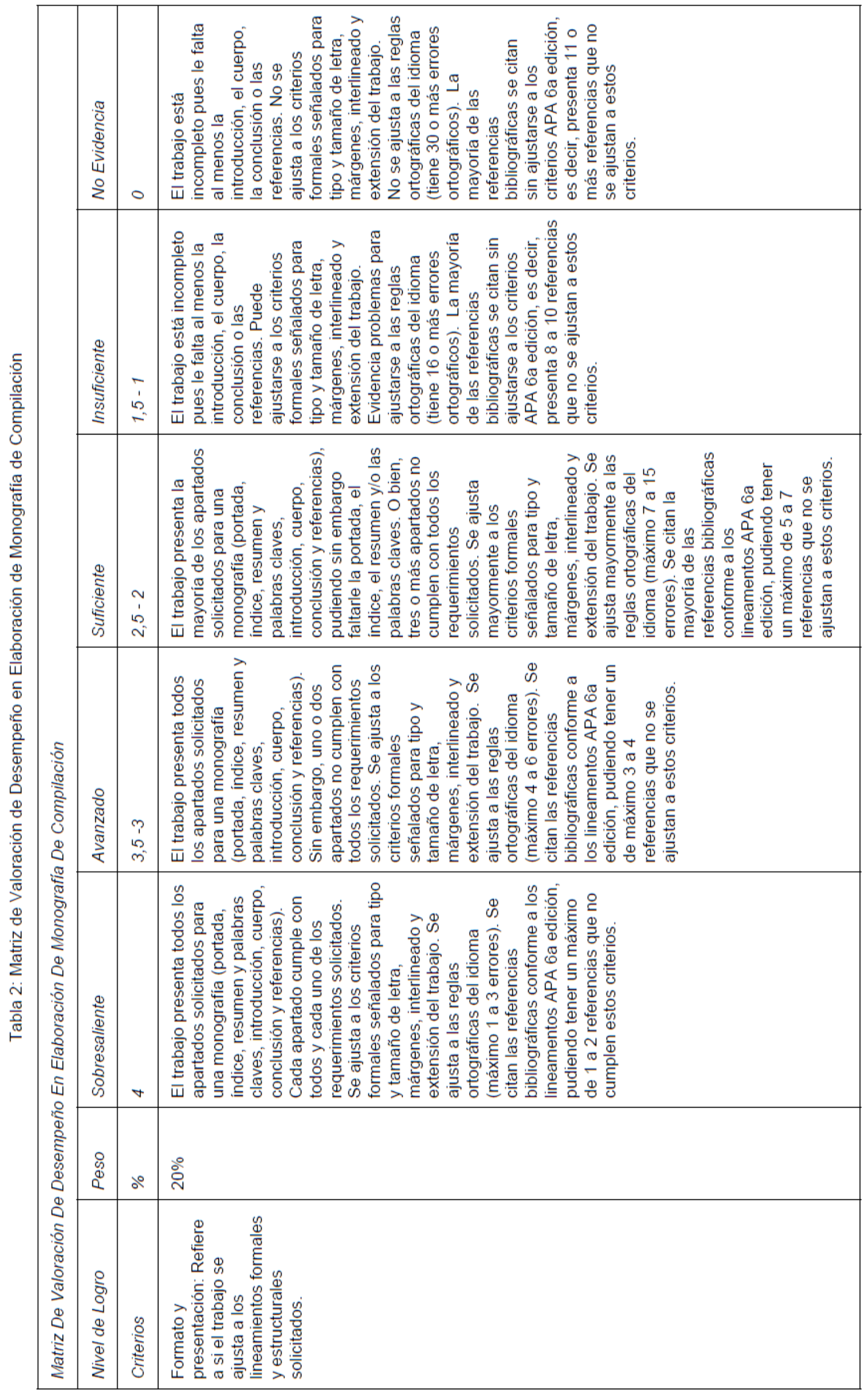




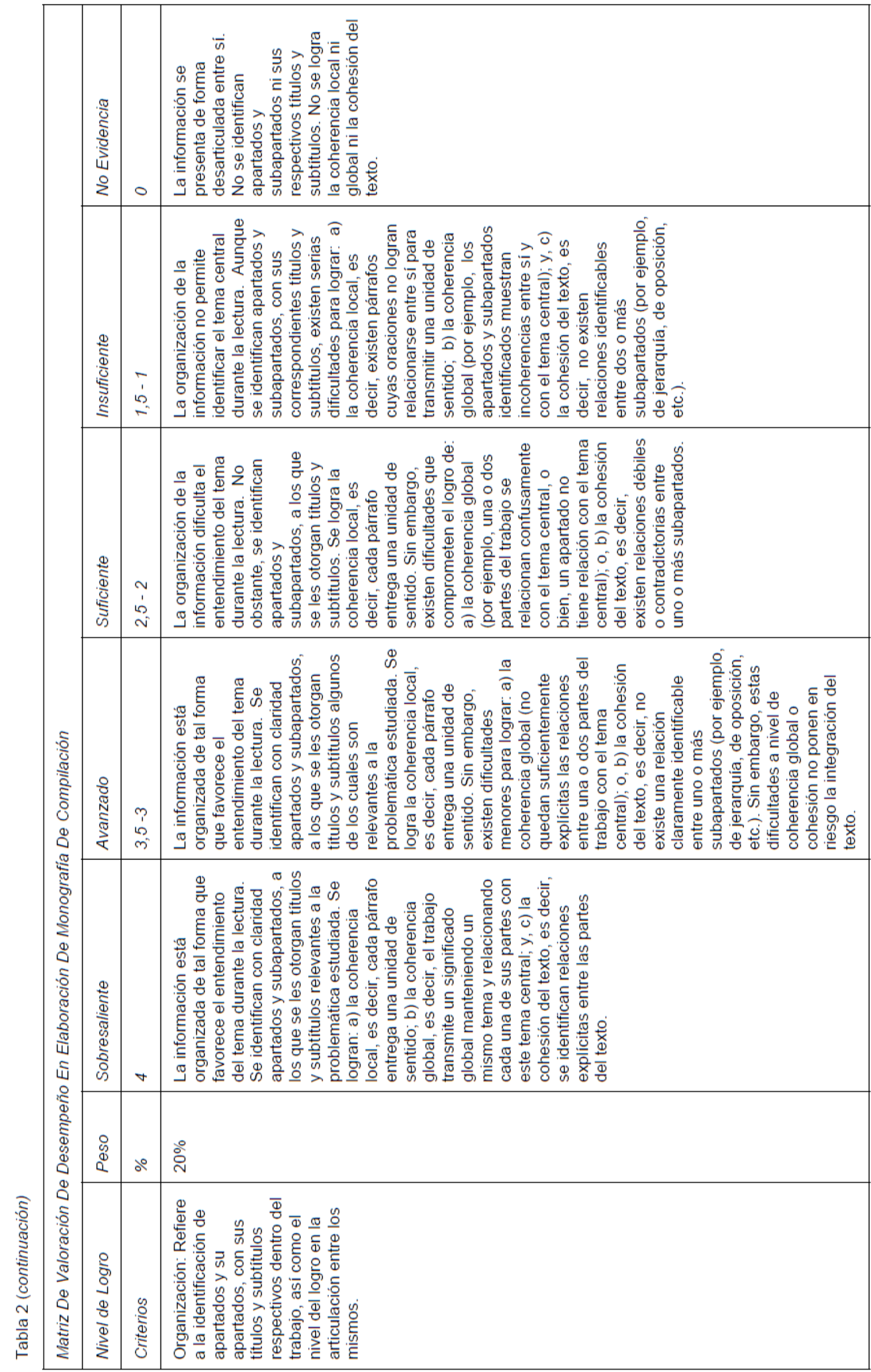




\begin{tabular}{|c|c|c|c|c|}
\hline & 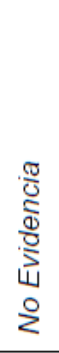 & 0 & 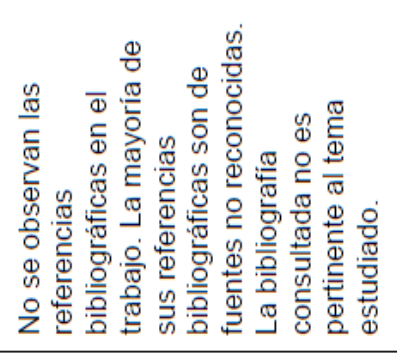 & 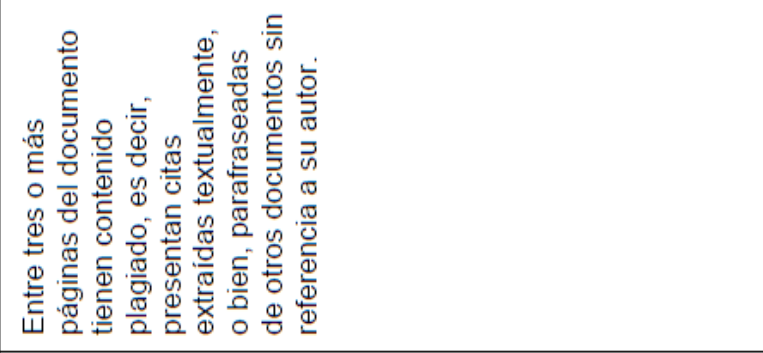 \\
\hline & 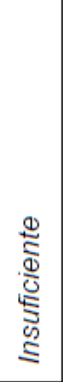 & & 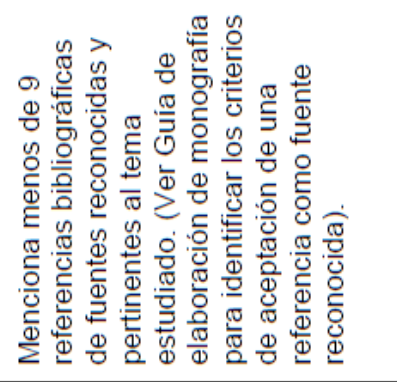 & 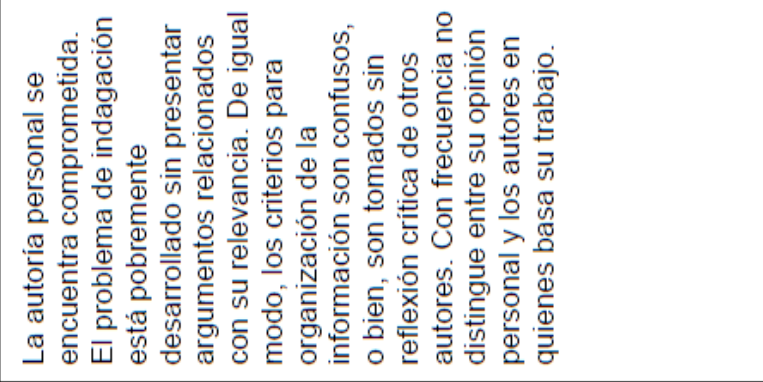 \\
\hline & 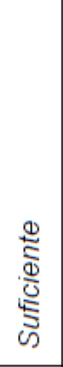 & & 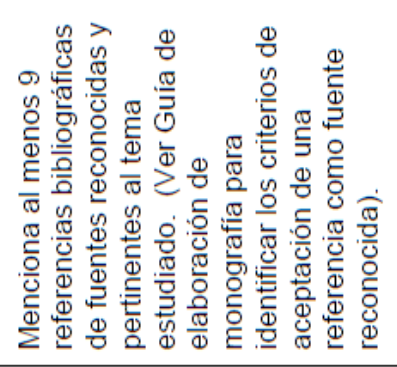 & 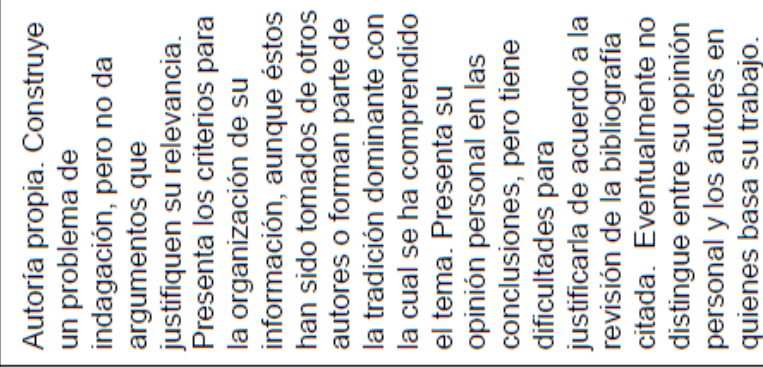 \\
\hline 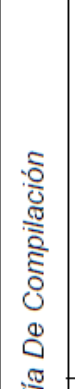 & 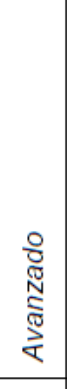 & & 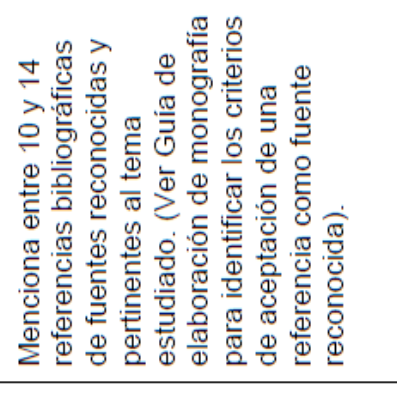 & 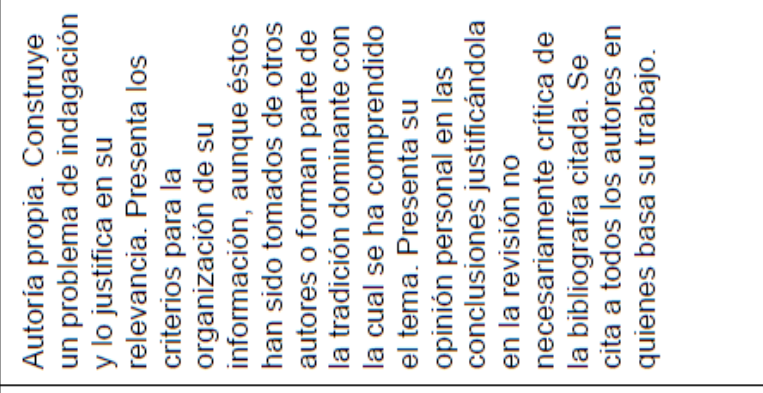 \\
\hline 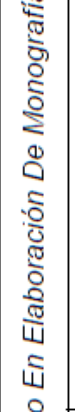 & 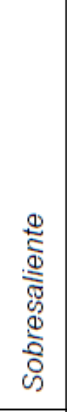 & $\forall$ & 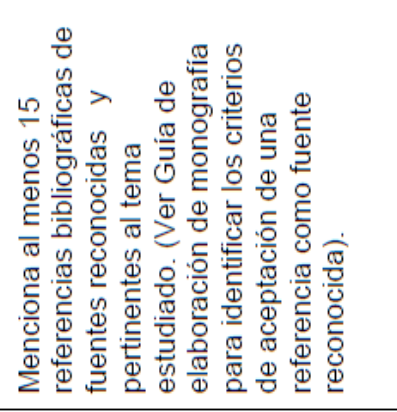 & 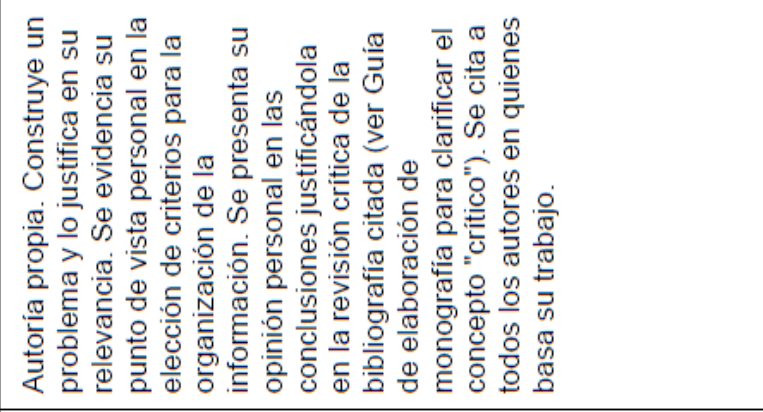 \\
\hline 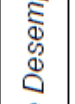 & $\begin{array}{l}8 \\
8 \\
0 \\
0\end{array}$ & dீ & ஓें & ㅇํㄹ \\
\hline 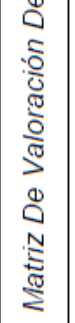 & 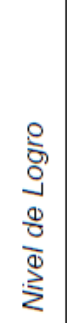 & 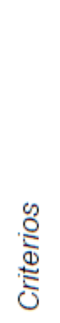 & 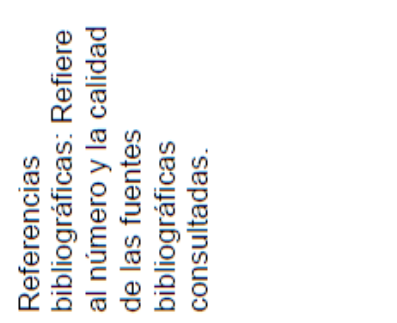 & 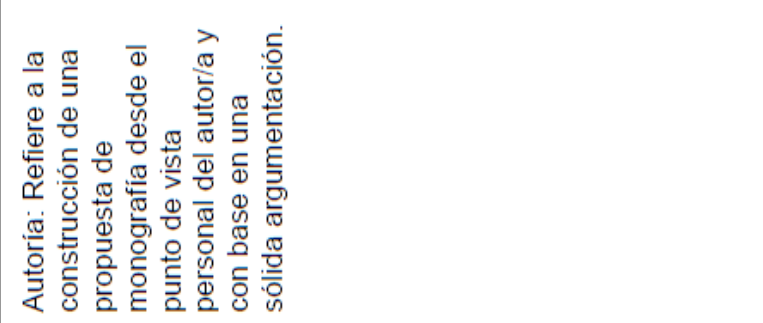 \\
\hline
\end{tabular}


Tabla 2 (continuación)

\begin{tabular}{|c|c|c|c|c|c|c|}
\hline $\begin{array}{l}\text { Nivel de } \\
\text { Logro }\end{array}$ & Peso & Sobresaliente & Avanzado & Suficiente & Insuficiente & No Evidencia \\
\hline Criterios & $\%$ & 4 & $3,5-3$ & $2,5-2$ & $1,5-1$ & 0 \\
\hline $\begin{array}{l}\text { Logro del } \\
\text { trabajo: } \\
\text { Refiere al } \\
\text { logro general } \\
\text { del producto } \\
\text { solicitado, } \\
\text { esto es, una } \\
\text { monografía } \\
\text { de } \\
\text { compilación }\end{array}$ & $15 \%$ & $\begin{array}{l}\text { El trabajo de } \\
\text { investigación } \\
\text { expone de } \\
\text { manera } \\
\text { exhaustiva y } \\
\text { organizada, la } \\
\text { revisión de un } \\
\text { tema particular en } \\
\text { el que se incluye } \\
\text { puntos de vista } \\
\text { personales y } \\
\text { críticos (ver Guía } \\
\text { de elaboración de } \\
\text { monografía para } \\
\text { clarificar el } \\
\text { concepto } \\
\text { "crítico"). Se } \\
\text { abordan las } \\
\text { principales teorías } \\
\text { explicativas de la } \\
\text { temática, } \\
\text { considerando el } \\
\text { alcance de éstas } \\
\text { y sus } \\
\text { correspondientes } \\
\text { autores. La } \\
\text { revisión se } \\
\text { organiza } \\
\text { considerando un } \\
\text { eje } \\
\text { problematizador } \\
\text { para la temática. }\end{array}$ & $\begin{array}{l}\text { El trabajo de } \\
\text { investigación } \\
\text { expone de } \\
\text { manera } \\
\text { exhaustiva y } \\
\text { organizada, la } \\
\text { revisión de un } \\
\text { tema particular en } \\
\text { el que se incluye } \\
\text { puntos de vista } \\
\text { personales. Se } \\
\text { abordan las } \\
\text { principales } \\
\text { teorías } \\
\text { explicativas de la } \\
\text { temática, } \\
\text { considerando el } \\
\text { alcance de éstas } \\
\text { y sus } \\
\text { correspondientes } \\
\text { autores. La } \\
\text { revisión se } \\
\text { organiza } \\
\text { considerando un } \\
\text { eje } \\
\text { problematizador } \\
\text { para la temática. }\end{array}$ & $\begin{array}{l}\text { El trabajo de } \\
\text { investigación } \\
\text { expone de } \\
\text { manera amplia y } \\
\text { organizada, la } \\
\text { revisión de un } \\
\text { tema particular } \\
\text { en el que se } \\
\text { incluye puntos } \\
\text { de vista } \\
\text { personales. Se } \\
\text { abordan la } \\
\text { mayoría de las } \\
\text { principales } \\
\text { teorías } \\
\text { explicativas de } \\
\text { la temática, } \\
\text { aunque hay } \\
\text { limitaciones en } \\
\text { la presentación } \\
\text { de sus alcances } \\
\text { y de sus } \\
\text { autores. La } \\
\text { revisión se } \\
\text { organiza } \\
\text { considerando un } \\
\text { eje } \\
\text { problematizador } \\
\text { para la temática. }\end{array}$ & $\begin{array}{l}\text { El trabajo de } \\
\text { investigación } \\
\text { expone de } \\
\text { manera limitada } \\
\text { y/o sin suficiente } \\
\text { organización, la } \\
\text { revisión de un } \\
\text { tema particular } \\
\text { en el que se } \\
\text { incluye puntos de } \\
\text { vista personales. } \\
\text { Puede abordar } \\
\text { una o dos de las } \\
\text { principales } \\
\text { teorías } \\
\text { explicativas en la } \\
\text { temática, pero lo } \\
\text { hace de forma } \\
\text { superficial sin } \\
\text { considerar los } \\
\text { alcances de las } \\
\text { teorías. La } \\
\text { revisión se } \\
\text { organiza sin que } \\
\text { se identifique un } \\
\text { eje articulador } \\
\text { para la temática. }\end{array}$ & $\begin{array}{l}\text { El trabajo de } \\
\text { investigación } \\
\text { expone de } \\
\text { manera in- } \\
\text { completa la } \\
\text { revisión de un } \\
\text { tema particular } \\
\text { sin que se } \\
\text { incluyan pun- } \\
\text { tos de vista } \\
\text { personales ni } \\
\text { críticos. Se } \\
\text { abordan } \\
\text { teorías y } \\
\text { autores cuyos } \\
\text { contenidos no } \\
\text { son pertinentes } \\
\text { a la temática } \\
\text { estudiada. La } \\
\text { revisión se } \\
\text { organiza sin } \\
\text { que se } \\
\text { identifique un } \\
\text { eje articulador } \\
\text { para la } \\
\text { temática. O } \\
\text { bien, presenta } \\
\text { un trabajo que } \\
\text { corresponde a } \\
\text { otro producto } \\
\text { de aprendizaje } \\
\text { (por ej. ensayo) }\end{array}$ \\
\hline
\end{tabular}

Tabla 3: Estadísticas de fiabilidad

\begin{tabular}{|c|c|c|}
\hline $\begin{array}{c}\text { Alfa de } \\
\text { Cronbach }\end{array}$ & $\begin{array}{c}\text { Alfa de Cronbach basada en } \\
\text { elementos estandarizados }\end{array}$ & $\begin{array}{c}\text { N de } \\
\text { elementos }\end{array}$ \\
\hline 0.832 & 0.847 & 5 \\
\hline
\end{tabular}

Sobre la confiabilidad de la matriz de valoración, la medida de coherencia interna utilizando el coeficiente Alfa de Cronbach muestra como resultado un valor de 0,832 lo que indica una alta consistencia o confiabilidad interna del instrumento, tal como se observa en la Tabla 3. El proceso de los datos en términos de cada ítem en particular se expresa en la Tabla 4.

El resultado relevante se puede observar en la columna final la cual muestra que la presencia de cada uno de los ítems es significativa en cuanto a la confiabilidad total del instrumento. Sólo en el caso de la Autoría se observa un aumento de la Confiabilidad total si se suprime este elemento, aunque la diferencia con el valor total $(0,832)$ es mínimo $(0,028)$. Todos los otros ítems muestran una baja de la confiabilidad si se suprimieran. Finalmente, se muestra el análisis respecto a la desviación estándar de cada componente y el nivel de discriminación de la matriz de valoración en la Tabla 5. Los resultados presentados en la Tabla 5 permiten observar que, sin considerar la ponderación de cada factor, la desviación estándar de cada componente es similar, es decir, en promedio todos los valores se distancian de la media de una manera semejante, correspondiendo a distribuciones homogéneas. Lo mismo puede observarse en relación al puntaje total ponderado. 
Tabla 4: Estadísticas de total de elemento

\begin{tabular}{|c|c|c|c|c|c|}
\hline & $\begin{array}{c}\text { Media de escala } \\
\text { si el elemento se } \\
\text { ha suprimido }\end{array}$ & $\begin{array}{c}\text { Varianza de } \\
\text { escala si el } \\
\text { elemento se ha } \\
\text { suprimido }\end{array}$ & $\begin{array}{c}\text { Correlación total } \\
\text { de elementos } \\
\text { corregida }\end{array}$ & $\begin{array}{c}\text { Correlación } \\
\text { múltiple al } \\
\text { cuadrado }\end{array}$ & $\begin{array}{c}\text { Alfa de Cronbach si el } \\
\text { elemento se ha } \\
\text { suprimido }\end{array}$ \\
\hline $\begin{array}{c}\text { Formato y } \\
\text { presentación }\end{array}$ & 7.949 & 16.347 & 0.549 & 0.407 & 0.822 \\
\hline Organización & 8.000 & 15.074 & 0.728 & 0.608 & 0.781 \\
\hline $\begin{array}{c}\text { Referencias } \\
\text { bibliográficas }\end{array}$ & 8.833 & 13.240 & 0.712 & 0.555 & 0.775 \\
\hline Autoría & 8.514 & 13.970 & 0.477 & 0.299 & 0.860 \\
\hline Logro del trabajo & 8.616 & 13.185 & 0.796 & 0.721 & 0.751 \\
\hline
\end{tabular}

Tabla 5: Número de sujetos, mínimo y máximo de puntaje, media, desviación estándar e índice de discriminación

\begin{tabular}{|l|r|r|r|r|r|r|}
\hline & $N$ & Mínimo & Máximo & Media & Desviación estándar & Índice Discriminante \\
\hline Formato y presentación & 69 & 1,0 & 4,0 & 2,522 &, 9642 & 0,36 \\
\hline Organización & 69 &, 0 & 4,0 & 2,478 &, 9756 & 1 \\
\hline Referencias bibliográficas & 69 &, 0 & 4,0 & 1,645 & 1,2837 & 0,35 \\
\hline Autoría & 69 &, 0 & 4,0 & 1,833 & 1,1333 & 0,44 \\
\hline Logro del trabajo & 69 &, 0 & 4,0 & 1,862 & 1,1971 & 0,77 \\
\hline Total100 & 69 &, 55 & 4,00 & 2,1007 &, 93117 & \\
\hline N válido (por lista) & 69 & & & & & \\
\hline
\end{tabular}

En relación al nivel de discriminación de la matriz de valoración, en la Tabla 5 se observa que todos los índices $\mathrm{D}$ son iguales o superiores a 0.35 y pueden ser considerados de buenos a excelentes bajo los criterios de Ebel y Frisbie (1986, en Escudero et al., 2000). Lo anterior muestra, por tanto, que la prueba posee un muy buen nivel de discriminación. Finalmente, en relación al nivel de dificultad de los factores evaluados en la matriz de valoración, se observa que los estudiantes mostraron un desempeño suficiente en Formato y presentación y Organización (Medias: 2,5 y 2,4 respectivamente) y un desempeño insuficiente en Logro del trabajo, Autoría y Referencias bibliográficas.

\section{DISCUSIÓN}

Con base en los resultados obtenidos se concluye que la matriz de valoración diseñada para la valoración de competencias de gestión del conocimiento y pensamiento crítico a través del desempeño del estudiante en una monografía de compilación, es válida y confiable. En este sentido, la monografía en tanto evidencia de aprendizaje basado en una investigación documental donde se debe organizar, integrar y valorar críticamente la información (Corona, 2015), permite observar el desempeño de los estudiantes en ambas competencias, en los niveles de logro definidos para cada una y conforme a lo esperable en estudiantes de primer año de pregrado de psicología. Considerando las limitaciones que con frecuencia enfrentan los docentes de los cursos de primer año para realizar evaluaciones cuando los grupos de estudiantes son numerosos, se considera de relevancia conocer las propiedades psicométricas de la matriz de valoración diseñada a la vez que se sugiere a los docentes de educación superior estimar su uso.

Según mostraron los resultados, los estudiantes lograron un desempeño suficiente en los criterios Formato y presentación y Organización, lo que puede relacionarse con el hecho de que la estructura del documento es un aspecto básico para las tareas académicas de primer año de pregrado. Por otra parte, el desempeño insuficiente en los criterios de Autoría y Logro del Trabajo sugiere que los estudiantes cuyos trabajos se evaluaron presentan dificultades para argumentar y realizar un análisis crítico entre varias ideas y 
posiciones de múltiples autores. En este sentido, sería conveniente reforzar el desarrollo de las competencias gestión del conocimiento y pensamiento crítico al menos en estudiantes de primer año de la carrera. Para ello, se podrían propiciar otras instancias de intervención para el uso de la matriz de valoración, entre ellas, analizar dicha herramienta con los estudiantes para que aprendan a usarla, lo que en otros estudios ha sido considerado como un factor que favorece los beneficios del uso de la rúbrica (Jones et al., 2016). Por último, específicamente con respecto a la competencia de pensamiento crítico habría que evaluar otro tipo de actividades que aporten a su desarrollo, entre otras, por ejemplo, la técnica de intervención socrática que favorece el desarrollo de habilidades como la inferencia, la explicación y la distinción entre puntos de vista (Betancourth et al; 2012).

\section{CONCLUSIONES}

La matriz de valoración diseñada evalúa de manera confiable y válida el logro de las competencias gestión del conocimiento y pensamiento crítico en estudiantes de pregrado en el ámbito de la formación en Epistemología de las Ciencias Sociales. Adicionalmente, la rúbrica mostró un Índice de Discriminación por cada criterio que va de bueno a excelente. Estas características psicométricas dan cierta garantía al docente respecto a que el instrumento puede ser usado para una evaluación consistente y útil a sus estudiantes. Por otra parte, debido a que en este estudio se optó por usar la consistencia interjuez o docente como medida de confiabilidad, se sugiere que este tipo de elección metodológica se complemente en futuros estudios con el uso de otros estadígrafos como el coeficiente de correlación de Pearson o el coeficiente de correlación intraclase a partir de los cuales realizar un análisis interjueces (D’Antoni et al., 2009; Gürsul y Keser, 2009). Por último, ya que en este estudio los estudiantes no fueron partícipes del proceso de valoración de la matriz, se sugiere que en otras investigaciones de este tipo se hagan parte del proceso, tal como sugieren Guzmán et al. (2012) y Gürsul y Keser (2009), lo que aumenta las posibilidades de que la matriz cumpla su objetivo de apoyo en el aprendizaje para cada estudiante.

\section{AGRADECIMIENTOS}

Esta investigación fue patrocinada por la línea Proyecto de Innovación Docente de la Universidad Católica de Temuco a través del proyecto 2014 titulado "Validez y confiabilidad de dos rúbricas utilizadas en los procesos de evaluación de aprendizaje profundo en estudiantes de primer y segundo año de Psicología".

\section{REFERENCIAS}

Atkinson, D. y S.L. Lim, Improving assessment process in Higher Education: Student and teacher perceptions of the effectiveness of a rubric embedded in a LMS, doi: 10.14742/ajet.526, Australasian Journal of Educational Technology, 29(5), 651-666 (2013)

Betancourth, S.; K. Insuasti y N. Riascos, Pensamiento crítico a través de la discusión socrática en estudiantes universitarios, https://goo.gl/rX6sxs, ISNN: 0124-5821, Revista Virtual Universidad Católica del Norte, 35, 47-167 (2012)

Biggs, J.; C. Tang, Teaching for quality Learning at University, 3a Ed., 50-63, Mc Graw Hill, New York, USA (2007)

Blanco, A., Desarrollo y Evaluación de Competencias en Educación Superior, 1a Ed., 17-27, Narcea S.A., Madrid, España (2011)

Corona, J.L., Uso e importancia de las monografías, https://goo.gl/fn1u5U, ISNN: 1561-3011, Revista Cubana de Investigaciones Biomédicas, 34(1), 64-68 (2015)

Corral, Y., Validez y confiabilidad de los instrumentos de investigación para la recolección de datos, https://goo.gl/zfXENC, ISNN: 1316-5917, Revista Ciencias de la Educación, 19(33), 228-247 (2009)

D’Antoni, A. V., G.P. Zipp y V.G. Olson, Interrater reliability of the mind map assessment rubric in a cohort of medical students, doi: 10.1186/1472-6920-9-19, BMC Medical Education, 9(19), 1-8 (2009)

Escudero, E.B., N.L. Reyna y M.R. Morales, Nivel de dificultad y poder de discriminación del Examen de Habilidades y Conocimientos Básicos (EXHCOBA), https://goo.gl/TMkddZ, ISNN: 1607-4041, Revista Electrónica de Investigación Educativa, 2(1), 10 -28 (2000)

Fernández, A., La evaluación orientada al aprendizaje en un modelo de formación por competencias en la educación universitaria. https://goo.gl/o48vFc; ISSN:1887-4592, Rev. de Docencia Univ.,8(1),11-34 (2010)

Gairín, J., Competencias para la gestión del conocimiento y el aprendizaje, https://goo.gl/9D4Rww, ISSN: 0210-0630, Cuadernos de Pedagogía, 370, 24-27 (2007) 
García-Ros, R., Diseño y utilización de rúbricas en la enseñanza universitaria, una aplicación en la titulación de Psicología, https://goo.gl/fQm4pT, ISSN: 1696-2095, Electronic Journal of Research in Educational Psychology, 10(3), 1477-1492 (2012)

Gatica, F. y T.N. Uribarren, ¿Cómo elaborar una rúbrica? https://goo.gl/HcYVQ7, ISSN: 2007-5057, Investigación en Educación Médica, 2 (1), 61-65 (2012)

Gürsul, F. y H. Keser, The effects of online and face to face problem based learning environments in mathematics education on student's academic achievement, doi: 10.1016/j.sbspro.2009.01.501, Procedia Social and Behavioral Sciences, 2817-2824 (2009)

Guzmán, Y.; R. Flores y F. Tirado, La evaluación de la competencia argumentativa en foros de discusión en línea a través de rúbricas, https://goo.gl/6G4A2n, ISNN: 1665-2673, Innovación Educativa, 12(60), 17-40 (2012)

Hernández, R.; C. Fernández y P. Batista, Metodología de la Investigación, 4ª Ed., 273 - 442. Mc Graw HIII, Ciudad de México, México (2008)

Jones, L.; B. Allen; P. Dunn y L. Brooker, Demystifying the rubrica five-step pedagogy to improve student understanding andutilisation of marking criteria, doi: 10.1080/07294360.20.16.1177000, Higher Education Research \& Development, 4360 (May), 1-14 (2016)

Lim, J.M., Rubric-referenced oral production assessments: perceptions on the use and actual use of rubrics in oral production assessments of high school students of St Scholastica's College, Manila, doi: 10.1186/2229-0443-3-4, Language Testing in Asia, 3, (4), 1-14 (2013)

Lu, J. y Z. Zhang, Assessing and Supporting Argumentation with Online Rubrics, doi:10.5539/ies.v6n7p66, International Education Studies, 6 (7), 66-77 (2013)

Manríquez, L., ¿Evaluación en competencias? doi: 10.4067/S0718-07052012000100022, Inf. Tecnol., 38 (1), 353-366 (2012)

Mora, G., Fundamentos Filosóficos de las Ciencias Sociales. Guía de Aprendizaje para el Estudiante de la Carrera de Psicología de la UC Temuco, Temuco-Chile (2015)

Muñoz, C. y Valenzuela, J., Características psicométricas de una rúbrica para evaluar expresión escrita a nivel universitario, doi: 10.4067/S0718-50062015000600010, Formación Universitaria, 8(6), 75-84 (2015)

Oviedo, H. y A. Campo, Aproximación al uso del coeficiente alfa de Cronbach, https://goo.gl/B9kMqs, ISSN: 0034-7450, Revista Colombiana de Psiquiatría, 34(4), 572-580 (2005)

Panadero, E.; J.A. Tapia y J.A. Huertas, Rubrics vs. self-assessment scripts: effects on first year university students' selfregulation and performance, doi:10.1080/02103702.2014.881655, Infancia y Aprendizaje, Journal for the Study of Education and Development, 37(1), 149-183 (2014)

Prins, F.J.; R de Kleijn, y J.V. Tartwijk, Students' use of a rubric for research theses, doi:

10.1080/02602938.2015.1085954, Assessment \& Evaluation Higher Education, 42(2), 128-150 (2015)

Raposo, M. y E. Martínez, La rúbrica en la enseñanza universitaria: un recurso para la tutoría de grupos de estudiantes, doi: 10.4067/S0718-50062011000400004, Formación Universitaria, 4(4), 19-28 (2011)

Reddy, Y.M. y H. Andrade, A review of rubric use in higher education, doi: http://dx.doi.org/10.1080/02602930902862859, Assessment \& Evaluation Higher Education, 35(4), 435-448 (2010)

Reimers, F.M y C.K. Chung, Enseñanza y Aprendizaje siglo XXI: Metas, políticas educativas y currículo en seis países, $1^{\text {a }}$ Ed., 11-29. Fondo de Cultura Económica, Ciudad de México, México (2016)

UC Temuco, Competencias Genéricas UC Temuco: Para la Formación Integral de Ciudadanos Socialmente Responsables, Dirección General de Docencia, Temuco-Chile (2008)

UC Temuco, Modelo Educativo UC Temuco: Principios y Lineamientos. Dirección General de Docencia, Temuco-Chile (2007)

Villarroel, V. y D. Bruna, Reflexiones en torno a las competencias genéricas en educación superior: Un desafío pendiente, doi: 10.5027, Psicoperspectiva, 13(1), 23-34 (2014) 
